\title{
Educação permanente com agentes comunitários de saúde para instrumentalização da visita domiciliar: relato de experiência
}

\author{
Permanent education with community \\ health agents to provide home visits: \\ an experience report
}

\section{Cheila Karei Siega' ${ }^{1}$ (1) Carine Vendruscolo ${ }^{2}$ (i) Elisangela Argenta Zanatta ${ }^{3}$ (1)}

\footnotetext{
${ }^{1}$ Autora para correspondência. Universidade do Estado de Santa Catarina (Chapecó). Santa Catarina, Brasil. cheilasiega@gmail.com 2,3Universidade do Estado de Santa Catarina (Chapecó). Santa Catarina, Brasil. carine.vendruscolo@udesc.br, elisangela.zanatta@udesc.br
}

\begin{abstract}
RESUMO | OBJETIVO: Relatar o processo de educação permanente com agentes comunitários de saúde, para instrumentalizá-los à Visita Domiciliar, por meio da reestruturação coletiva de um Roteiro, à luz da Promoção da Saúde. MÉTODO: Estudo descritivo, tipo relato de experiência, desenvolvido em um município do Meio Oeste catarinense entre os meses de novembro e dezembro de 2017. Participaram atores em uma área assistida pela Estratégia Saúde da Família que representam o ensino, a gestão, a atenção e o controle social, em quatro encontros orientados por Círculos de Cultura. RESULTADOS: Foi reestruturado e validado um Roteiro para a Visita Domiciliar, explorandose a temática da Promoção da Saúde, como tecnologia para utilização dos agentes comunitários de saúde. A ação fomentou a consciência crítica e a autonomia dos envolvidos. CONSIDERAÇõES FINAIS: Houve um efetivo movimento de educação permanente, tendo o agente comunitário de saúde como protagonista. Destaca-se os Círculos de Cultura como uma estratégia facilitadora do processo, cujo resultado foi a construção coletiva de uma tecnologia que, certamente, irá contribuir para a reorientação do serviço, na perspectiva da Promoção da Saúde.
\end{abstract}

DESCRITORES: Atenção Primária à Saúde. Educação Continuada. Agentes Comunitários de Saúde. Visita Domiciliar.

\begin{abstract}
OBJECTIVE: To report the process of permanent education with community health agents to give them instruments for Home Visits, through collective restructure of a Script, in the light of Health Promotion. METHOD: Descriptive study, experience report type, developed in a municipality in the Midwest of Santa Catarina between the months of November and December 2017. Participants participated in an area assisted by the Family Health Strategy that represent teaching, management, care and control in four meetings guided by Culture Circles. RESULTS: A Script for Home Visits was restructured and validated, exploring the theme of Health Promotion, as a technology for the use of community health agents. The action fostered critical awareness and the autonomy of those involved. FINAL CONSIDERATIONS: There was an effective permanet education movement, having the community health agent as a protagonist. We highlight that Culture Circles were an strategy that facilitated the process because the result was a collective construction of a technology that, certainly, will contribute to reorient the service, in the perspective of Health Promotion.
\end{abstract}

DESCRIPTORS: Primary health care. Continuing education. Community health agents. Home visit. 


\section{Introdução}

A Atenção Primária à Saúde (APS), no Brasil denominada Atenção Básica $(A B)$, é a principal porta de entrada para a Rede que compõe o Sistema único de Saúde (SUS), o qual contribui para a determinação constitucional de que a saúde é um direito fundamental do ser humano garantido pelo Estado'.

A Política Nacional da Atenção Básica (PNAB) ressalta que esse ponto da Rede de Atenção à Saúde (RAS) oferece um conjunto de ações de saúde individuais, familiares e coletivas que envolvem diferentes aspectos do cuidado, desenvolvidos por meio de práticas integradas e gestão qualificada. Essas ações são realizadas por uma equipe multidisciplinar, dirigidas a uma população em território definido, tendo sua expansão, consolidação e qualificação realizada por meio da Estratégia Saúde Família (ESF). Assim, a AB, centro de comunicação da RAS, é coordenadora do cuidado e ordenadora das ações e serviços disponibilizados e opera como estratégia para o cuidado integral e direcionado as necessidades de saúde da população'.

Com tais contornos, a $A B$ visa superar o modelo de atenção centrado na cura das doenças e desenvolver ações de Promoção da Saúde de acordo com os preceitos do SUS. Para tanto, a Política Nacional de Promoção da Saúde (PNPS) ${ }^{2}$ vem reafirmando as estratégias da Carta de Otawa $(1986)^{3}$, sinalizando para o conceito de saúde como resultado de uma série de determinantes e levando em conta a felicidade das pessoas, como um dos requisitos para tal. A Promoção da Saúde é expressa por meio de um conjunto de valores como: equidade, justiça social, ética, autonomia dos indivíduos, dentre outros, sendo necessárias condições e recursos fundamentais para sua implementação ${ }^{2}$. Ao encontro dessa discussão, a Carta de Ottawa apresenta cinco campos de ação para a Promoção da Saúde: políticas públicas saudáveis, ambientes favoráveis a saúde, ação comunitária, habilidades pessoais e reorientação dos serviços de saúde ${ }^{4}$.

Destaca-se no presente estudo, a reorientação dos serviços de saúde, campo de ação que propõe abolir o modelo biomédico, centrado nas doenças e na sua cura, para buscar concepções de Promoção da Saúde, por meio de transformações na organização do financiamento, nas práticas e na formação profissional ${ }^{3}$. A Educação Permanente em Saúde (EPS) apresenta-se, nesse contexto, como uma estratégia para mudança na concepção e nas práticas dos atores que operam nas Equipes de Saúde da Família (EqSF), mediante um processo de aprendizagem centrado no mundo do trabalho, em que o aprender e ensinar incorporam-se ao cotidiano das equipes ${ }^{5}$. Os pressupostos da EPS se fundamentam no protagonismo dos sujeitos envolvidos, por meio da sua intervenção crítica na realidade, a partir da aprendizagem significativa, expressa por experiências anteriores. Ao substituírem a postura de expectadores pela de protagonistas, os sujeitos reconhecem sua autonomia e assumem responsabilidades, comprometem-se e desenvolvem o senso de pertencimento ${ }^{6}$.

Sob este enfoque, em processos de EPS, é recomendável a participação dos sujeitos que representam o "prisma da educação em saúde", quais sejam: representantes do ensino, do controle social, da gestão e da atenção, considerando os reflexos que os atores provocam nesses segmentos, com intuito de mobilizar processos interativos e de ação na realidade?.

O "prisma" é uma metáfora que amplia o ideário do quadrilátero da formação ${ }^{8}$, em que os sujeitos e segmentos representados na saúde - educadores, usuários, gestores e trabalhadores - estão implicados em realidades que repercutem na maneira de exercer essa representação e, por conseguinte, deliberam sobre as mais variadas situações ${ }^{7}$. As relações de integração ensino-serviço, no quadrilátero da formação, são determinadas pelo nível de envolvimento dos diferentes sujeitos e instâncias envolvidas no processo ${ }^{8}$. Por meio do "prisma" (que amplia a ideia do quadrilátero) é possível romper com os espaços instituídos de cada sujeito social do quadrilátero, a fim de constituir redes de atenção, nas quais cada ator se implique e constitua relações, nas quais cuidado, gestão e educação se interconectam? .

O agente comunitário de saúde (ACS) atua na EqSF, representando o segmento atenção. $\mathrm{Na}$ equipe, é o ACS que mantém contato direto com as pessoas da comunidade mediante visitas periódicas às famílias, tendo assim, papel fundamental ao estar intimamente ligado à população tornando-se o articulador dos processos de trabalho da equipe, por conhecer bem a comunidade e ter maior facilidade de acesso ao território ${ }^{10}$.

Seu principal instrumento de trabalho, a Visita Domiciliar (VD), pode ser compreendida a partir da realização de etapas: planejamento, execução, registro das informações e avaliação do processo, tendo 
como principais ações o desenvolvimento de orientações que devem priorizar ações que contemplem a Promoção da Saúde ${ }^{11}$. A VD pode ser tratada como uma tecnologia não material, pois, por meio da educação em saúde, possibilita a transformação da realidade, mediante construção de vínculo entre profissionais e usuários, atendendo a longitudinalidade do cuidado (atributo essencial da APS/AB) ${ }^{12}$. Nesse sentido, o que se observa é a dificuldade das EqSF e, por conseguinte, dos ACS, para realizar a VD de forma estruturada, desperdiçando assim, oportunidades de promover a saúde dos indivíduos e comunidade.

O ideário da EPS posiciona o trabalhador como produtor e reprodutor de conhecimento e de mudanças na práxis, pois no cotidiano se depara com situações-problema e precisa encontrar respostas e soluções ${ }^{7}$. Para produzir saúde, é necessário dar visibilidade as experiências vivenciadas no mundo do trabalho e dialogar sobre elas, na perspectiva da Promoção da Saúde.

O objetivo deste artigo foi relatar o processo de educação permanente com agentes comunitários de saúde, para instrumentalizá-los à Visita Domiciliar, por meio da reestruturação coletiva de um Roteiro, à luz da Promoção da Saúde.

\section{Método}

Estudo descritivo, do tipo relato de experiência, sobre uma proposta de intervenção que surgiu a partir da disciplina "Promoção da Saúde ao Indivíduo e Coletividades", ministrada no Curso de Mestrado Profissional em Enfermagem na na Atenção Primária à Saúde da Universidade do Estado de Santa Catarina (UDESC), em que a mestranda, com função de Enfermeira no local de intervenção, identificou a necessidade de instrumentalização dos ACS para a VD.

A proposta contou com a participação de 20 trabalhadores, membros da EqSF, tendo como protagonista o ACS. A participação dos atores seguiu o seguinte critério para inclusão: ser integrante do "prisma da educação em saúde"7. Assim, foram convidados, por meio de Ofício, representantes de todos os segmentos. 0 segmento ensino foi representado pela mestranda, coordenadora das atividades; a gestão foi representada pela Coordenadora de AB do município; a atenção foi representada por profissionais de uma EqSF, em uma Unidade Básica de Saúde (UBS), totalizando:
11 ACS, um Cirurgião Dentista, um Auxiliar de Saúde Bucal, uma Enfermeira, três Técnicos de Enfermagem e dois Médicos; e a comunidade representada por três líderes comunitários dos bairros pertencentes à UBS. A atividade foi desenvolvida em um município da Região Meio Oeste do estado de Santa Catarina, entre os meses de novembro e dezembro de 2017.

O desenvolvimento da ação percorreu o seguinte caminho metodológico: elaboração do projeto de intervenção; apresentação em sala de aula aos colegas e professores da disciplina "Promoção da Saúde ao Indivíduo e Coletividades", a fim de qualificar a proposta; desenvolvimento das atividades propostas na UBS e território adscrito. Para a efetivação da proposta de intervenção foram realizadas quatro ações de EPS, aproveitando-se as reuniões de equipe realizadas pela EqSF. Essas ações se desenvolveram no formato de Círculos de Cultura (CC), seguindo o Método de Paulo Freire, composto por três fases: 1) Investigação Temática; 2) Codificação e Descodificação e, 3) Desvelamento Crítico ${ }^{4}$. Em cada encontro, as discussões foram devidamente registradas, por meio de anotações realizadas pela pesquisadora, a partir do consentimento dos participantes.

Nos CC, os sujeitos tornam-se ativos e participantes, por meio de debates, diálogos e construção de saber, buscando uma reflexão crítica sobre situações da realidade vivida - situação existencial limite, que são obstáculos, barreiras, empecilhos ou dificuldades, que interferem na vida dos indivíduos e que precisam ser superados ${ }^{4}$.

O CC é um espaço dialógico no qual a troca de saberes acontece de maneira natural e ganham destaque o respeito e a ética entre os envolvidos. O diálogo é guiado a partir dos Temas Geradores (TG), na medida em que acontecem os encontros. Logo no primeiro encontro, emergiu o TG: "dificuldade para realização de VD, devidamente estruturada". Foi a partir dessa temática (posteriormente, codificada e desvelada) que se desenvolveram os encontros subsequentes. Durante as etapas de codificação e descodificação, foram refletidos mais a fundo os desdobramentos do TG, levantando-se as potencialidades e dificuldades encontradas no processo de VD. No desvelamento crítico, os participantes compreenderam suas fragilidades e propuseram ações para otimizar a VD, o que culminou na reestruturação do roteiro de VD, posteriormente validado pelos ACS e pela EqSF. 
O estudo aqui apresentado obteve aprovação pelo Comitê de Ética em Pesquisa da Universidade Federal de Santa Catarina sob parecer $n^{\circ}$ 2.448.665 e CAAE: 79506717.6.3001.0121.

\section{Resultados}

O quadro 1 ilustra, de forma resumida, como foi problematizado o TG: "dificuldade para realização de VD, devidamente estruturada", a partir das etapas do Método Freireano, ocorridas nos quatro encontros (Círculos de Cultura - CC):

Quadro 1. Desenvolvimento do TG: "dificuldade para realização de VD, devidamente estruturada”, segundo etapas do Método Freireano e encontros realizados

\begin{tabular}{|l|l|l|}
\hline \multicolumn{1}{|c|}{ Encontros / Etapas do Método } & \multicolumn{1}{|c|}{ Atividades Desenvolvidas } & \multicolumn{1}{c|}{ Resultados } \\
\hline CC 1 - Investigação Temática & $\begin{array}{l}\text { - Apresentação do projeto à equipe; } \\
\text { - Discussão do roteiro de VD; } \\
\text { - Sinalização das facilidades e } \\
\text { dificuldades do roteiro existente. }\end{array}$ & $\begin{array}{l}\text { - Identificação do TG; } \\
\text { - Identificação de problemas } \\
\text { relacionados à VD. }\end{array}$ \\
\hline $\begin{array}{l}\text { CC 2 - Codificação e } \\
\text { Descodificação }\end{array}$ & $\begin{array}{l}\text { - Problematização do TG, a partir de } \\
\text { diálogos, à luz da Promoção da } \\
\text { Saúde. }\end{array}$ & $\begin{array}{l}\text { - Elaboração de um mapa com } \\
\text { alternativas para superação dos } \\
\text { problemas identificados. }\end{array}$ \\
\hline CC 3 - Desvelamento Crítico & $\begin{array}{l}\text { - Problematização do TG, a partir de } \\
\text { diálogos e registro de novas ideias. }\end{array}$ & $\begin{array}{l}\text { - Reestruturação/qualificação do } \\
\text { roteiro de VD. }\end{array}$ \\
\hline CC 4 - Validação do roteiro de VD & $\begin{array}{l}\text { - Compreensão das fragilidades da } \\
\text { equipe e agrupamento de ideias } \\
\text { para otimizar o processo de VD. }\end{array}$ & - Roteiro de VD validado. \\
\hline
\end{tabular}

Fonte: Os autores (2019).

A fim de valorizar o Método Freireano como ação educativa e libertadora, os resultados serão apresentados no contexto da sua produção, durante os CC.

No $1^{\circ} \mathrm{CC}$, foi realizada a apresentação do projeto para a equipe. Após, buscou-se identificar o TG a partir dos principais problemas relacionados à VD. Foi elencado o TG: "dificuldade para realização de VD, devidamente estruturada". Por meio do diálogo com os presentes sobre o roteiro de VD existente, foram sinalizadas as facilidades e dificuldades encontradas para a sua aplicação.

O TG se desdobrou em subtemas, na forma de situações, as quais foram descritas em um quadro, destacando-se como principais facilidades: boa receptividade em algumas moradias, principalmente onde residem idosos. Como dificuldades encontradas pelos ACS, foi sinalizado: verificar as carteiras de vacina das crianças e da gestante, levar a equipe de saúde para VD de puerpério até o $7^{\circ}$ dia do Recém-Nascido, participação dos hipertensos e diabéticos no grupo de Educação em Saúde, encontrar as pessoas em suas residências em horário comercial.

No $2^{\circ} \mathrm{CC}$, foram utilizadas tarjetas, nas quais os participantes escreveram atividades que designavam como de prevenção de doenças e promoção da saúde. Ficou clara a dificuldade conceitual sobre o significado de cada uma das expressões, as quais, por vezes, se confundiam. Após, apresentou-se a temática da Promoção da Saúde, por meio do diálogo sobre os pressupostos da Carta de Ottawa e da PNPS. Na sequência, voltou-se aos temas que foram referidos como dificuldades e buscou-se, coletivamente, alternativas para superá-los, mediante diálogo entre os participantes. 
$\mathrm{O} 3^{\circ} \mathrm{CC}$ foi marcado pela apresentação do roteiro existente, problematizado entre os participantes, a fim de levantar sugestões de melhoria/adequação dos temas já abordados. A partir das discussões, surgiram novas ideias que serviram para reestruturar e qualificar o roteiro de VD dos ACS com vistas à Promoção da Saúde.

No CC, foi desenhada uma árvore em papel pardo, na qual a raiz representou o roteiro de VD atual e os galhos e folhas representaram as novas percepções e sugestões que emergiram do diálogo. Ao final, o objetivo proposto foi atingido, pois ocorreu a reestruturação do roteiro, com a participação colaborativa, ficando definido esse modelo para utilização pelo ACS, a partir de então.

Antes da realização do $4^{\circ} \mathrm{CC}$, o roteiro reestruturado foi utilizado durante o período de cinco dias pelos ACS, durante a realização das VD, servindo como base para suas ações em cada abordagem realizada às famílias, de acordo com a realidade encontrada. Durante o último encontro, foi evidenciado pelos ACS que o material reestruturado apresentou uma forma mais didática para guiar suas ações, pois as ilustrações e a disposição da escrita facilitaram que recordassem os temas a serem abordados em cada situação. A validação do conteúdo do roteiro ocorreu neste último $\mathrm{CC}$, realizado pela EqSF, sendo que não foram consideradas necessárias adequações naquele momento. Poderão surgir novas possibilidades, as quais, conforme acordado, serão incluídas no roteiro para assim, qualificar as VD desenvolvidas, de maneira permanente e a partir das necessidades que emergirem da prática laboral.

\section{Discussão}

O Método Freireano permitiu um nível de envolvimento dos atores, que foi determinante para a construção de relações fecundas entre eles, resultando em um processo efetivo de EPS, sobretudo pela representação de vários segmentos que atuam no campo da saúde, com seus diferentes pontos de vista sobre o processo de trabalho ${ }^{13}$.

A primeira fase do Método, a Investigação Temática, consiste no momento em que ocorre a descoberta do universo das palavras, vocabulários ou situações e temas extraídos do cotidiano dos participantes ${ }^{5}$. O TG que emergiu deste momento, representou um assunto com grande significado para o grupo e que fez parte do processo educativo ${ }^{9}$. Normalmente, o TG emerge das situações limite, as quais devem ser conhecidas pelos indivíduos, possibilitando que nelas se reconheçam, na medida em que se apresentam 4 .

O processo de Codificação e Descodificação possibilita que se busquem nos TG seus significados, a fim de contextualizá-los, ampliando o conhecimento e a tomada de consciência sobre as discussões ${ }^{4}$. A partir das situações limite elencadas, as codificações devem ser simples e oferecer várias possibilidades na sua descodificação, abrindo-se na direção de outros temas ${ }^{4}$. Foi neste momento que se resgatou a Carta de Ottawa, documento revisado em 2014, que destaca a importância dos fatores condicionantes e determinantes da saúde. A Carta tem como pressupostos a intra e a intersetorialidade e a criação de redes de corresponsabilidade que buscam a melhoria da qualidade de vida, embora recomende, também, cuidados voltados à diminuição de riscos e à prevenção de doenças ${ }^{14}$. A discussão do TG à luz deste material possibilitou que os participantes refletissem sobre o seu ideário em relação à Promoção da Saúde, oferecendo subsídios para a revisão do roteiro de VD. Este momento da ação educativa também representou a realidade e as possibilidades que se apresentam a partir dela, e fomentou um novo olhar sobre as atribuições dos ACS no que diz respeito as ações de Promoção da Saúde durante as VD.

No último encontro, Desvelamento Crítico, houve a representação da realidade, mediante as possibilidades e a análise dos conteúdos extraídos da codificação ${ }^{4}$. Foi neste momento que se discutiu o conceito de Tecnologia, compreendida como criação humana para resolver problemas práticos. Com essa intencionalidade, se espera que a Tecnologia contribua positivamente, como instrumento de trabalho para a obtenção de melhores resultados. Nota-se a importância de o ACS utilizar instrumentos que possam guiar suas atividades nas VD, a fim de aproximar a equipe aos indivíduos, famílias e coletividades e transformar suas ações cotidianas em um trabalho sistematizado e resolutivo. O roteiro de VD, bem como o planejamento de ações cotidianas de EPS entre a equipe, pode qualificar o trabalho e produzir resultados positivos ${ }^{11}$. 
Nessa direção, instrumentos construídos coletivamente, validados pela equipe de trabalho, e principalmente, com a participação de todos os envolvidos no processo, podem ser efetivos meios tecnológicos para o alcance do que se deseja modificar. Assim, compreendeu-se o roteiro de VD como uma Tecnologia a serviço dos $A C S$, no âmbito da $A B^{15}$.

Em relação à Promoção da Saúde e sua potencialidade para a reorientação dos serviços na $A B$, constam entre os seus princípios na PNPS'2, a participação social e a autonomia, relacionadas à inclusão de pontos de vista dos indivíduos, grupos e coletividades na identificação de problemas e soluções e na valorização de potencialidades, com vistas a fomentar a corresponsabilidade no planejamento, execução e avaliação de ações.

Nessa perspectiva, o movimento desencadeado nesta experiência, de fato, contribuiu para a reorientação de parte do trabalho da equipe, representado pela VD. Por meio do diálogo entre os sujeitos do prisma da educação em saúde, movimentos desta ordem pressupõem a participação efetiva, de todos os segmentos, o que contribui para um bom resultado. Entretanto, apesar de promover a autonomia e a corresponsabilização do ACS junto à EqSF, houve uma adesão e participação incipientes, por parte dos segmentos gestão e controle social, sobretudo nos momentos que conformaram a intervenção propriamente dita. Tal situação corrobora estudo que identificou a participação comunitária como prática pouco evidenciada entre as iniciativas de trabalho intersetorial no âmbito da $A B$, na perspectiva da Promoção da Saúde ${ }^{16}$.

A ciência só é possível quando se aborda o objeto investigado por meio de técnicas, a partir de um método apoiado em fundamentos epistemológicos. Dessa forma, os CC, como estratégica metodológica para a produção e análise das informações, representou uma potencialidade, como forma de aprofundar o tema e provocar a transformação do processo de trabalho da EqSF ${ }^{17}$.

\section{Considerações finais}

O estudo permitiu a realização de um processo de educação permanente com ACS, a fim de instrumentalizá-los à VD, por meio da reestruturação coletiva de um Roteiro, considerado por eles como uma Tecnologia a seu serviço. Para além do cumprimento do objetivo, vale destacar que houve a criação de vínculos entre pesquisadores e participantes, devido ao fato de que todos se identificaram com o TG e se envolveram na busca por soluções para o seu problema, num efetivo movimento de EPS.

Os CC foram importantes no sentido de dar voz a este sujeito, o ACS, no âmbito da EqSF, valorizando seu trabalho e suas experiências, além de (co)responsabilizá-lo pela produção e implementação de Tecnologias que impactem na Promoção da Saúde e, por conseguinte, na resolubilidade da AB.

Como limitação do estudo, destaca-se a incipiente participação dos segmentos gestão e controle social nos CC. Nesse sentido, espera-se que movimentos de EPS com participação dos sujeitos que compõem o prisma façam parte da rotina dessa EqSF e que o presente estudo se apresente como propulsor de ideias para outras equipes que tenham como missão o fortalecimento da $A B$ e do SUS.

Por fim, sugere-se novos estudos, com ampliação de cenários e perspectiva longitudinal para melhor compreender o processo de trabalho do ACS e das EqSF, a partir da utilização de tecnologias não materiais.

Considera-se que a metodologia dos CC, adotada e detalhada nessa intervenção, possa subsidiar outras EqSF para a realização de adequações nos seus instrumentos de trabalho, de acordo com cada realidade.

\section{Contribuições das autoras}

Siega CK participou da concepção, delineamento, análise, interpretação dos dados e redação do artigo. Vendruscolo $\mathrm{C}$ e Zanatta EA participaram da concepção, delineamento, análise, interpretação dos dados, redação e revisão crítica do artigo. 


\section{Conflitos de interesses}

Nenhum conflito financeiro, legal ou político envolvendo terceiros (governo, empresas e fundações privadas, etc.) foi declarado para nenhum aspecto do trabalho submetido (incluindo, mas não se limitando a subvenções e financiamentos, participação em conselho consultivo, desenho de estudo, preparação de manuscrito, análise estatística, etc.).

\section{Referências}

1. Brasil. Ministério da Saúde. Portaria $n^{\circ} 2.436$, de 21 de setembro de 2017. Aprova a Política Nacional de Atenção Básica, estabelecendo a revisão de diretrizes para a organização da Atenção Básica, no âmbito do Sistema Único de Saúde (SUS) [Internet]. 2017 [acesso em 2017 nov 16]. Disponível em: http://bvsms.saude.gov.br/bvs/saudelegis/gm/2017/ prt2436_22_09_2017.html

2. Brasil. Ministério da Saúde. Portaria $n^{\circ} 2.446$, de 11 de novembro de 2014. Redefine a Política Nacional de Promoção da Saúde (PNPS). Diário Oficial [da] União. 2014 nov 13;68 seção 1.

3. World Health Organization. The Ottawa charter for health promotion. Ottawa: World Health Organization; 1986.

4. Heidemann ITSB, Wosny AM, Boehs AE. Promoção da Saúde na Atenção Básica: estudo baseado no método Paulo Freire. Ciênc. saúde coletiva. 2014;19(8):3553-59. doi: 10.1590/141381232014198.11342013

5. Pinheiro DGM, Scabar TG, Maeda ST, Fracolli LA, Pelicioni MCF, Chiesa AM. Competências em promoção da saúde: desafios da formação. Saude soc. 2015;24(1):180-88. doi: 10.1590/S010412902015000100014

6. Cypriano CC, Marçal CCB, Heidmann ITSB. Pensar e refletir para um novo agir pedagógico em saúde. In: Prado ML, Schmidt KR. Paulo Freire: a boniteza de ensinar e aprender na saúde. Florianópolis: NFR/UFSC; 2016. p.119-132.

7. Vendruscolo C, Prado ML, Kleba ME. Reorientação do ensino no SUS, para além do quadrilátero, o prisma da educação. Revista Reflexão e Ação. 2016;24(3):246-60. doi: 10.17058/rea.v24i3.5420

8. Ceccim RB, Feuerwerker LCM. O quadrilátero da formação para a área da saúde: ensino, gestão, atenção e controle social. Physis: revista de saúde coletiva. 2004;14(1):41-65. doi: 10.1590/S010373312004000100004

9. Freire P. Educação como prática de liberdade. 40.ed. Rio de Janeiro: Paz e Terra; 2017.
10. Alonso CMC, Béguin PD, Duarte FJCM. Trabalho dos agentes comunitários de saúde na Estratégia Saúde da Família: metassíntese. Rev. Saúde Pública. 2018;52:14. doi: 10.11606/ s1518-8787.2018052000395

11. Magalhães KA, Giacomin KC, Santos WJ, Firmo JOA. A visita domiciliária do agente comunitário de saúde a famílias com idosos frágeis. Ciência \& Saúde Coletiva. 2015;20(12):3787-96. doi: 10.1590/1413-812320152012.07622014

12. Starfield B. Atenção primária: equilíbrio entre necessidades de saúde, serviços e tecnologia. Brasília: UNESCO, Ministério da Saúde; 2002.

13. Vendruscolo C, Trindade LL, Kleba ME, Prado ML. Rethinking the Health Care Model through the reorientation of training. Rev Bras Enferm. 2018;71(suppl 4):1674-82. doi: 10.1590/0034-71672017-0055

14. Carvalho FFB, Cohen SC, Akerman M. Refletindo sobre o instituído na promoção da saúde para problematizar 'dogmas'. Saúde Debate. 2017;41(3):265-76. doi: 10.1590/0103110420175320

15. Soratto J, Pires DEP, Dornelles S, Lorenzetti J. Family Health Strategy: a technological innovation in health. Text Context Nursing. 2015;24(2):584-92. doi: 10.1590/0104$\underline{07072015001572014}$

16. Prado NMBL, Santos AM. Promoção da saúde na Atenção Primária à Saúde: sistematização de desafios e estratégias intersetoriais. Saúde Debate. 2018;42(1):379-95. doi: 10.1590/0103-11042018S126

17. Adamy EK, Zocche DAA, Vendruscolo C, Santos JLG, Almeida MA. Validation in grounded theory: conversation circles as a methodological strategy. Rev Bras Enferm. 2018;71(6):3299-304. doi: 10.1590/0034-7167-2017-0488 